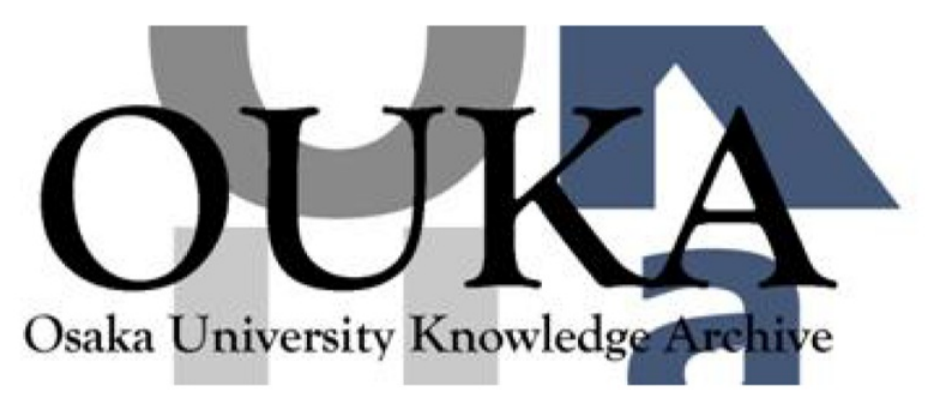

\begin{tabular}{|c|l|}
\hline Title & $\begin{array}{l}\text { One-pot electrochemical formation of meso, meso- } \\
\text { linked porphyrin arrays }\end{array}$ \\
\hline Author(s) & $\begin{array}{l}\text { Ogawa, Takuji; Nishimoto, Yoshihiro; Ono, } \\
\text { Noboru et al. }\end{array}$ \\
\hline Citation & CHEMICAL COMMUNICATIONS. 3 p.337-p. 338 \\
\hline Issue Date & 1998-02-07 \\
\hline oaire:version & VoR \\
\hline URL & https://hdl.handle.net/11094/3271 \\
\hline rights & \\
\hline Note & \\
\hline
\end{tabular}

Osaka University Knowledge Archive : OUKA

https://ir. Library. osaka-u. ac. jp/

Osaka University 


\title{
One-pot electrochemical formation of meso,meso-linked porphyrin arrays
}

\author{
Takuji Ogawa, ${ }^{* a}$ Yoshihiro Nishimoto, ${ }^{b}$ Naoya Yoshida, ${ }^{c}$ Noboru Ono ${ }^{b}$ and Atsuhiro Osukua*c \\ a Institute for Fundamental Research of Organic Chemistry, Kyushu University, Hakozaki, Higashi-ku, Fukuoka 812-81, Japan \\ ${ }^{b}$ Department of Chemistry, Faculty of Science, Ehime University, Matsuyama 790, Japan \\ c Department of Chemistry, Graduate School of Science, Kyoto University, Sakyo-ku, Kyoto 606-01, Japan
}

Electrochemical oxidation of [5,15-bis(3,5-di-tert-butylphenyl)porphyrinato]zinc(II) $1, \quad[5,15$-bis(4-methoxycarbonylphenyl)porphyrinato]zinc(II), $\quad[5,15$-bis(pentafluorophenyl)porphyrinato]zinc(II) or [10-bromo-5,15diphenylporphyrinato]zinc(II) in benzonitrile using a platinum net as the working electrode afforded the corresponding meso,meso-linked oligomer porphyrin arrays, and up to the octamer porphyrin array could be isolated for 1 .

Polynuclear porphyrins have been attracting considerable attention as biomimetic models of photosynthetic systems as well as photonic materials and functional molecular devices. ${ }^{1-7}$

In a previous communication we reported the oxidative coupling reaction of [5,15-bis(3,5-di-tert-butylphenyl)porphyrinato]zinc(II) 1 with silver(I) salts to give the porphyrin dimer 2 , the porphyrin trimer 3 and the porphyrin tetramer $4 .^{8}$ The possible mechanism proposed is an initial one-electron oxidation of zinc porphyrin with $\mathrm{Ag}^{\mathrm{I}}$ followed by nucleophilic attack of a neutral zinc porphyrin. This mechanism suggests that electrochemical oxidation also may lead to the formation of the meso,meso-coupled porphyrin arrays. Here we report an electrochemical preparation of the porphyrin arrays, by which the usage of expensive silver salts is not required, the oxidizing potentials can be best tuned to the porphyrin used, and the long porphyrin arrays can be prepared in a one-step procedure through prolonged electrolysis.

Porphyrin $1(n=1)(219 \mathrm{mg}, 0.293 \mathrm{mmol})$ was electrolyzed in dry benzonitrile $(210 \mathrm{ml})$ in a single cell with $\mathrm{NBu}_{4} \mathrm{ClO}_{4}(0.1$ M) as the electrolyte, a platinum net $(1.8 \times 2.4 \mathrm{~cm})$ as the counter electrode, and a platinum net $(3 \times 4 \mathrm{~cm})$ as the working electrode; the electricity used was $0.732 \mathrm{mF}$. The porphyrin products were purified by being passed through an alumina and then a silica gel chromatography column, and isolated by preparative scale HPLC using a gel permeation column (GPCHPLC). The structures were identified through comparison with the authentic samples: ${ }^{8} \mathbf{1}(44.0 \%), \mathbf{2}(n=2)(20.7 \%), \mathbf{3}(n=3)$ $(2.9 \%)$ and $\mathbf{4}(n=4)(0.9 \%)$. When porphyrin $1(211 \mathrm{mg}, 0.282$ mmol) was electrolyzed under similar conditions using electricity of $1.65 \mathrm{mF}$, the higher homologous pentamer $\mathbf{5}(n=5)$, hexamer $6(n=6)$, heptamer $7(n=7)$ and octamer $8(n=8)$ could be isolated at yields of $0.5,0.4,0.2$ and $0.05 \%$, respectively, together with 1 (6.7\%), 2 (10.2\%), 3 (3.2\%) and 4 $(1.6 \%)$ by GPC-HPLC. The new product was characterized by ${ }^{1} \mathrm{H}$ NMR spectroscopy (500 or $600 \mathrm{MHz}$ ) and FAB mass spectrometry or MALDI-TOF mass spectrometry. $\dagger$

Treatment of [5,15-bis(4-methoxycarbonylphenyl)porphyrinato]zinc(II) 9 at higher oxidation potential $(+0.68 \mathrm{~V} v s$. Ag/ $\left.\mathrm{AgNO}_{3}\right)$ than $1\left(+0.54 \mathrm{~V}\right.$ vs. $\left.\mathrm{Ag} / \mathrm{AgNO}_{3}\right)$ with $\mathrm{AgClO}_{4}$ under the conditions reported previously ${ }^{8}$ did not afford the corresponding meso,meso-linked porphyrin arrays, although an unidentified complex mixture together with $c a$. $60 \%$ of the recovered starting material were obtained. However, when 9 (51 $\mathrm{mg}, 79 \mu \mathrm{mol}$ ) was electrolyzed using electricity of $134 \mu \mathrm{F}$, it afforded the meso,meso-linked porphyrin dimer $\mathbf{1 0}(34.3 \%)$, porphyrin trimer $11(6.9 \%)$ and the starting porphyrin 9 $(47.4 \%)$. Similarly, [5,15-bis(pentafluorophenyl)porphyrinato]zinc(II) $12\left(+0.77 \mathrm{~V}\right.$ vs. $\left.\mathrm{Ag} / \mathrm{AgNO}_{3}\right)$ and (10-bromo5,15-diphenylporphyrinato)zinc(II) $\quad \mathbf{1 3} \quad(+0.56 \quad \mathrm{~V} \quad$ vs. $\mathrm{Ag} /$ $\mathrm{AgNO}_{3}$ ), which did not give the corresponding meso,meso-linked porphyrin arrays by the silver salt method, afforded the corresponding dimers $\mathbf{1 4}$ and $\mathbf{1 5}$ under similar conditions by electrolysis in isolated yields of 11.6 and $47.5 \%$, respectively. Thus the electrochemical method has a wider scope for the substrates than the silver salt method.

These meso,meso-linked porphyrin arrays so far isolated were quite soluble in $\mathrm{CHCl}_{3}$. Of particular note is the fact that even the arrays of porphyrin 9, 12 and 13, which have little sterical hindrance to prevent $\pi-\pi$ stacking of the porphyrin planes, also had high solubility. This is probably because the porphyrin planes connect nearly perpendicular to each other due to their sterical requirement, and hence their intermolecular $\pi-\pi$ stacking was largely prevented.

These results indicate that the present electrochemical method is a convenient way of preparing meso,meso-linked porphyrin arrays which should be good candidates for long 'molecular wires' ${ }^{\prime}, 10$ having a linear rigid structure and high solubility.

Work at Kyoto was partly supported by Grants-in Aid for Scientific Research (No. 08874074) from the Ministry of Education, Science, Sports and Culture of Japan and by the Tokuyama Science Foundation.

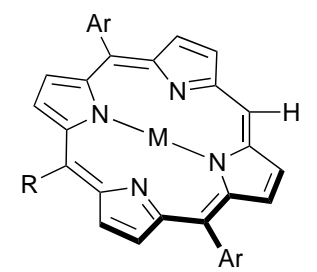

1; $\mathrm{Ar}=3,5$-di-tert-butylphenyl, $\mathrm{R}=\mathrm{H}$

9; $\mathrm{Ar}=4$-methoxycarbonylphenyl, $\mathrm{R}=\mathrm{H}$

12; $\mathrm{Ar}=$ pentafluorophenyl, $\mathrm{R}=\mathrm{H}$

13; $\mathrm{Ar}=$ phenyl, $\mathrm{R}=\mathrm{Br}$

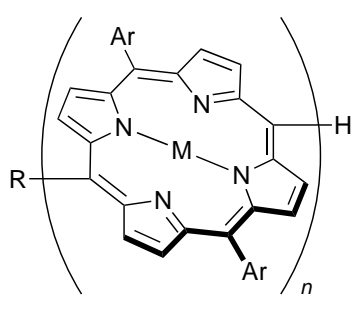

2; $n=2$ 3; $n=3 \quad 4 ; n=4 \quad 5 ; n=5 \quad 6 ; n=6 \quad 7 ; n=7 \quad 8 ; n=8$

$10 ; n=211 ; n=3$

$14 ; n=2$

$15 ; n=2$

Scheme 1 Reagents and conditions: i, Anodic oxidation, $\mathrm{Pt}$ net as working electrode, $\mathrm{Pt}$ net as counter electrode, in benzonitrile, $0.1 \mathrm{M} \mathrm{NBu}_{4} \mathrm{ClO}_{4}$ 


\section{Notes and References}

* E-mail: ogawat@ms.ifoc.kyushu-u.ac.jp

$\dagger 5(\mathrm{Ar}=3,5$-di-tert-butylphenyl, $\mathrm{R}=\mathrm{H}, n=5): \delta_{\mathrm{H}}\left(\mathrm{CDCl}_{3}\right) 1.37\left(\mathrm{~s}, \mathrm{Bu}{ }^{\mathrm{t}}\right)$, $1.40\left(\mathrm{~s}, \mathrm{Bu}^{\mathrm{t}}\right), 1.50(\mathrm{~s}, \mathrm{Bu}), 7.62(\mathrm{t}, J 2.0,4 \mathrm{H}, \mathrm{Ar}-4-\mathrm{H}), 7.65(\mathrm{t}, J 1.5,2 \mathrm{H}$, Ar-4-H), 7.76 (t, J 2.0, 4 H, Ar-4-H), 8.12 (d, J 2.0, 8 H, Ar-2,6-H), 8.15 (d, $J$ 1.5, $4 \mathrm{H}, \mathrm{Ar}-2,6-\mathrm{H}), 8.16(\mathrm{~d}, J 2,8 \mathrm{H}, \mathrm{Ar}-2,6-\mathrm{H}) 8.19$ (d, $J 4.5,4 \mathrm{H}, \beta$ protons), 8.32 (d, $J 4.5,4 \mathrm{H}, \beta$ protons), 8.35 (d, $J 5.0,4 \mathrm{H}, \beta$ protons), 8.36 (d, J 5.0, $4 \mathrm{H}, \beta$ protons), 8.75 (d, J 5.0, $4 \mathrm{H}, \beta$ protons), $8.82(\mathrm{~d}, J 4.5,4 \mathrm{H}$, $\beta$ protons), 8.84 (d, $J 4.0,4 \mathrm{H}, \beta$ protons), 8.85 (d, J 4.5, $4 \mathrm{H}, \beta$ protons), 9.22 (d, J 4.5, $4 \mathrm{H}, \beta$ protons), 9.53 (d, J 4.5, $4 \mathrm{H}, \beta$ protons) and $10.42(\mathrm{~s}, 2 \mathrm{H})$; $m / z(F A B) 3742.1\left(\mathrm{C}_{240} \mathrm{H}_{252} \mathrm{~N}_{20} \mathrm{Zn}_{5}\right.$ requires 3743.7). $6(\mathrm{Ar}=3,5$-di-tertbutylphenyl, $\mathrm{R}=\mathrm{H}, n=6): \mathrm{m} / z$ (TOF) $4563\left(\mathrm{C}_{288} \mathrm{H}_{302} \mathrm{~N}_{24} \mathrm{Zn}_{6}\right.$ requires 4492). 7 ( $\mathrm{Ar}=3,5$-di-tert-butylphenyl, $\mathrm{R}=\mathrm{H}, n=7): \mathrm{m} / z$ (TOF) 5237 $\left(\mathrm{C}_{336} \mathrm{H}_{352} \mathrm{~N}_{28} \mathrm{Zn}_{7}\right.$ requires 5240). $8(\mathrm{Ar}=3,5$-di-tert-butylphenyl, $\mathrm{R}=\mathrm{H}$, $n=8): m / z$ (TOF) $6053\left(\mathrm{C}_{384} \mathrm{H}_{402} \mathrm{~N}_{32} \mathrm{Zn}_{8}\right.$ requires 5989). $10(\mathrm{Ar}=4-$ methoxycarbonylphenyl, $\mathrm{R}=\mathrm{H}, n=2): \delta_{\mathrm{H}}\left(\mathrm{CDCl}_{3}\right) 3.89(\mathrm{~s}, 12 \mathrm{H}), 8.03(\mathrm{~d}$, $4 \mathrm{H}, J$ 5.0, $\beta$ protons), 8.21 (d, $8 \mathrm{H}, J 8.5$, phenyl), 8.25 (d, $8 \mathrm{H}, J 8.5$, phenyl), 8.57 (d, $4 \mathrm{H}, J 4.5, \beta$ protons), 9.03 (d, $4 \mathrm{H}, J 4.5, \beta$ protons), 9.45 (d, $4 \mathrm{H}, \mathrm{J} 4.5, \beta$ protons) and 10.35 (s, $2 \mathrm{H}$, meso protons); $\mathrm{m} / \mathrm{z}$ (FAB) 1281.1 $\left(\mathrm{C}_{72} \mathrm{H}_{46} \mathrm{~N}_{8} \mathrm{O}_{8} \mathrm{Zn}_{2}\right.$ requires 1282.0). $11(\mathrm{Ar}=$ 4-methoxycarbonylphenyl, $\mathrm{R}=\mathrm{H}, n=3): \delta_{\mathrm{H}}\left[\left(\mathrm{CD}_{3}\right)_{2} \mathrm{CO}\right] 3.90\left(\mathrm{~s}, 6 \mathrm{H}\right.$, inner $\left.\mathrm{CH}_{3} \mathrm{OCOC}_{6} \mathrm{H}_{4}\right), 4.04(\mathrm{~s}$, $12 \mathrm{H}$, outer $\left.\mathrm{CH}_{3} \mathrm{OCOC}_{6} \mathrm{H}_{4}\right), 8.11(\mathrm{~d}, 4 \mathrm{H}, J 4.5, \beta$ protons $), 8.23(\mathrm{~d}, 4 \mathrm{H}, J$ $4.5, \beta$ protons $), 8.25-8.45\left(\mathrm{~m}, 24 \mathrm{H}, \mathrm{CH}_{3} \mathrm{OCOC}_{6} \mathrm{H}_{4}\right), 8.57$ (d, $4 \mathrm{H}, J 5.0, \beta$ protons), 8.65 (d, $4 \mathrm{H}, J 4.5$, $\beta$ protons), 9.05 (d, $4 \mathrm{H}, J 4.5, \beta$ protons), 9.58 (d, $4 \mathrm{H}, J 4.0, \beta$ protons) and 10.48 (s, $2 \mathrm{H}$, meso protons); $\mathrm{m} / \mathrm{z}$ (FAB) 1921.7 $\left(\mathrm{C}_{108} \mathrm{H}_{68} \mathrm{~N}_{12} \mathrm{O}_{12} \mathrm{Zn}_{3}\right.$ requires 1921.9). $14(\mathrm{Ar}=$ pentafluorophenyl, $\mathrm{R}=\mathrm{H}$, $n=2): \delta_{\mathrm{H}}\left(\mathrm{CDCl}_{3}\right) 8.18(\mathrm{~d}, 4 \mathrm{H}, J 4.5, \beta$ protons $), 8.65(\mathrm{~d}, 4 \mathrm{H}, J 5, \beta$ protons), 9.11 (d, $4 \mathrm{H}, J 4.5, \beta$ protons), 9.61 (d, $4 \mathrm{H}, J 5, \beta$ protons) and 10.50 (s, $2 \mathrm{H}$, meso protons); $m / z$ (FAB) $1410\left(\mathrm{C}_{64} \mathrm{H}_{18} \mathrm{~F}_{20} \mathrm{~N}_{8} \mathrm{Zn}_{2}\right.$ requires
1410). $15(\mathrm{Ar}=\mathrm{Ph}, \mathrm{R}=\mathrm{Br}, n=2): \delta_{\mathrm{H}}\left(\mathrm{CDCl}_{3}\right) 7.5-7.7(\mathrm{~m}, 12 \mathrm{H}, \mathrm{Ph}), 8.06$ (d, $4 \mathrm{H}, J 5, \beta$ protons), 8.19 (dd, $8 \mathrm{H}, J 8$ and $2, \mathrm{Ph}), 8.60(\mathrm{~d}, 4 \mathrm{H}, J 4.5, \beta$ protons), 9.04 (d, $4 \mathrm{H}, J 5$, protons) and $9.85(\mathrm{~d}, 4 \mathrm{H}, J 4.5, \beta$ protons $) ; m / z$ (FAB) $1207.7\left(\mathrm{C}_{64} \mathrm{H}_{36} \mathrm{Br}_{2} \mathrm{~N}_{8} \mathrm{Zn}_{2}\right.$ requires 1208.0).

1 M. O. Senge, W. W. Kalisch and K. Ruhlandt-Senge, Chem. Commun., 1996, 2149.

2 L. Ruhlmann and A. Giraudeau, Chem. Commun., 1996, 2007.

3 A. Giraudeau, L. Ruhlmann, L. E. Kahef and M. Gross, J. Am. Chem. Soc., 1996, 118, 2969.

4 A. Osuka, S. Marumo, N. Mataga, S. Taniguchi, T. Okada, I. Yamazaki, Y. Nishimura, T. Ohno and K. Nozaki, J. Am. Chem. Soc., 1996, 118, 155.

5 A. P. H. Schenning, F. B. G. Benneker, H. P. M. Geurts, X. Y. Liu and R. J. M. Nolte, J. Am. Chem. Soc., 1996, 118, 8549.

6 K. Susumu, T. Shimidzu, K. Tanaka and H. Segawa, Tetrahedron Lett., 1996, 37, 8399.

7 A. Osuka, S. Nakajima, T. Okada, S. Taniguchi, K. Nozaki, T. Ohno, I. Yamazaki, Y. Nishimura and N. Mataga, Angew. Chem., Int. Ed. Engl., 1996, 35, 92.

8 A. Osuka and H. Shimidzu, Angew. Chem., Int. Ed. Engl., 1997, 36, 135. Similar meso,meso-coupled porphyrin arrays have been prepared independently by different methods. Ref. 6 and R. G. Khoury, L. Jaquinod and K. M. Smith, Chem. Commun., 1997, 1057.

9 J. S. Schumm, D. L. Pearson and J. M. Tour, Angew. Chem., Int. Ed. Engl., 1994, 33, 1360.

10 J. M. Tour, Chem. Rev., 1996, 96, 537.

Received in Cambridge, UK, 23rd October 1997; 7/07653C 